\title{
Simplified Step-by-Step Nonlinear Static Program Investigating Equilibrium Conditions of Electrons in Atom and Ionization Energies: Case Study on Argon
}

\author{
Panagis G. Papadopoulos ${ }^{1}$, Christopher G. Koutitas ${ }^{2}$, Yannis N. Dimitropoulos ${ }^{3}$, \\ Elias C. Aifantis $4,5,6,7,8$
}

\begin{abstract}
${ }^{1}$ Structural Engineering Division, Civil Engineering Department, Aristotle University of Thessaloniki, Thessaloniki, Greece ${ }^{2}$ Fluid Mechanics Division, Civil Engineering Department, Aristotle University of Thessaloniki, Thessaloniki, Greece ${ }^{3}$ Physical Chemistry Division, Chemistry Department, University of Ioannina, Ioannina, Greece

${ }^{4}$ Aristotle University of Thessaloniki, Thessaloniki, Greece

${ }^{5}$ Michigan Technological University, Houghton, MI, USA

${ }^{6}$ Beijing University of Civil Engineering and Architecture, Beijing, China

${ }^{7}$ ITMO University, St. Petersburg, Russia

${ }^{8}$ Togliatti State University, Togliatti, Russia

Email: panaggpapad@yahoo.gr
\end{abstract}

How to cite this paper: Papadopoulos, P.G., Koutitas, C.G., Dimitropoulos, Y.N. and Aifantis, E.C. (2018) Simplified Step-by-Step Nonlinear Static Program Investigating Equilibrium Conditions of Electrons in Atom and Ionization Energies: Case Study on Argon. Open Journal of Physical Chemistry, 8, 33-56. https://doi.org/10.4236/ojpc.2018.82003

Received: March 29, 2018

Accepted: May 13, 2018

Published: May 16, 2018

Copyright (c) 2018 by authors and Scientific Research Publishing Inc. This work is licensed under the Creative Commons Attribution International License (CC BY 4.0).

http://creativecommons.org/licenses/by/4.0/

(c) (i) Open Access

\begin{abstract}
For investigation of equilibrium conditions of electrons in an atom, and Ionization Energies of Elements, a simplified deterministic static model is proposed. The electrons are initially uniformly and sparsely arranged on the outer surface of nucleus. Then, by taking into account the nucleus-electron interaction (attractive and repulsive) and the mutual electron-electron repulsions, and by a simple step-by-step nonlinear static analysis program, all the electrons are found to equilibrate on the outer surface of the same sphere, which is concentric and larger than nucleus. In a second stage, starting from an equilibrium sphere of electrons, one of the electrons is subjected to gradual forced removal, radially and outwards with respect to nucleus. Within each removal step, the produced work increment is determined and the increments are summed. When no more significant attraction is exerted by nucleus to removed electron, the total work gives the Ionization Energy. After removing of single electron, the remaining electrons fall on a lower shell, that is, they equilibrate on the outer surface of a smaller concentric sphere. For nucleus-electron interaction, an L-J (Lennard-Jones) type curve, attractive and repulsive, is adopted. When the parameter of this curve is $n>1.0$, the Ionization Energy exhibits an upper bound. As parameter $n$ increases from 1.0 up to 2.0 , the attractive potential of L-J curve is gradually weakened. The proposed
\end{abstract}


model is applied on Argon. It is observed that, as the number of electrons increases, the radius of equilibrium sphere increases, too, whereas the attractive nucleus-electron potential is reduced; thus the Ionization Energy is reduced, too. Particularly, as the number of electrons and the radius of equilibrium sphere exceed some critical values, the above two last quantities exhibit abrupt falls. A regular polyhedron is revealed, which can accommodate Elements up to atomic number $Z=146$, that is 28 more than $Z=118$ of existing last Element, as guide for initial locations of electrons in the above first program.

\section{Keywords}

Ionization Energy, Electrostatic Laws, Lennard-Jones Curve, Incremental Nonlinear Static Analysis, Atomic Radius, Rhombic Dodecahedron, Regular Polyhedron

\section{Introduction}

Aim of present work is to develop a simplified deterministic static model investigating the equilibrium conditions of electrons in an atom and the Ionization Energies of the Elements [1] [2].

The concept of Quantum Mechanics, used in Computational Chemistry, is stochastic; so, it is accurate, but complicated [3]-[8]. Recently, efforts have been made to use alternatively the Finite Element Method [9] [10]. For the moment, the proposed F.E.M models seem somewhat complicated, too.

It is recognized that a symmetric deterministic model can give results similar to those of a corresponding stochastic model [11] [12]. Such a symmetric deterministic model is proposed here.

The electron-electron electrostatic repulsion and the nucleus-electron interaction (attractive and repulsive), described by a Lennard-Jones type curve [13], are taken into account. And a simple step-by-step nonlinear static analysis algorithm is used [14].

Main finding of the proposed model is that all the electrons equilibrate on the outer surface of the same sphere, concentric and larger than nucleus. When one electron is removed from an equilibrium sphere, the remaining electrons fall to a lower shell, that is, they equilibrate on the outer surface of a sphere concentric and smaller than the previous equilibrium sphere and larger than nucleus.

The proposed model is applied on Argon [15], the noble gas of the third Period of Periodic Table [16], because, only for the first three Periods, complete data exist for Atomic Radii and Ionization Energies of Elements [17] [18] [19], for calibration and comparison.

Based on the results of the Application, observations are made on the variations, compared to each other, of main quantities of present problem: number of electrons, radius of equilibrium sphere of electrons, attractive potential of Lennard-Jones curve, Ionization Energy. 


\section{Proposed Model}

\subsection{Estimation of Equilibrium Positions of Electrons by Use of a Step-by-Step Nonlinear Static Program}

The surroundings of an atom are assumed as a dielectric medium, where the electrostatic laws hold. The nucleus is assumed motionless.

In order to find the equilibrium positions of electrons in an atom, the electrons are initially located on the outer surface (assumed spherical) of the nucleus, as shown in Figure 1(a), by using as guide a rhombic dodecahedron [20], which will be described in Section 2.5. The electrons are initially uniformly and sparsely arranged, so that to avoid very small distances between neighboring electrons, which create numerical problems.

Then, by taking into account nucleus-electron interactions (attractive and repulsive), described by a Lennard-Jones curve [13], which will be described in Section 2.4, and mutual electron-electron repulsions, as shown in Figure 1(b), and by using a simple and short computer program [14], described by the flow-chart of Figure 2, we find that all the electrons reach in equilibrium on the outer surface of the same sphere, concentric and larger than nucleus, as shown in Figure 1(c).

According to the flow-chart of Figure 2, the proposed computer program of step-by-step nonlinear static analysis for finding the equilibrium positions of electrons, in an atom, is as follows:

First, the general input data are read, which are: the atomic number $Z$ of the Element, equal to the number of its protons [1], the nuclear radius, given by the formula $r_{n}=r_{H} \sqrt[3]{Z}$, where $r_{H}=37 \mathrm{pm}$ nuclear radius of hydrogen [1], which is reasonable for assumption of homogeneous spherical nuclei. The number $v$ of electrons and the steplength $\Delta u$ of the algorithm, e.g. $\Delta u=0.001 \mathrm{pm}$, for which information will be given in Section 2.4.

Then, the initial locations $\bar{r}_{i}=\left\{x_{i} y_{i} z_{i}\right\}, i=1, \cdots, v$ of the electrons are read. In every step of the algorithm, as shown in Figure 2, for every electron $i$, first its distance $r$, from nucleus center $\mathrm{O}$ is determined and, by calling subroutine NUCLEL, the force $\bar{F}_{i}$ (attractive or repulsive), by which the nucleus acts on the electron, is found.

Then, the distance $r_{i k}$ of electron $i$ from every other electron $k$ is determined and, by calling the subroutine ELEL, the repulsive force $\overline{F_{i k}}$, by which electron $k$ acts on electron $i$ is found.

The forces $\overline{F_{i k}}$, from all the other electrons $k$, on the electron $i$ are summed to its force $\bar{F}_{i}$ from nucleus and the resultant force $\bar{F}_{i}$ on any electron $i$ is determined.

If the maximum absolute value of the out-of-balance forces of all the electrons is greater than the desired accuracy, e.g. if $\max \left|F_{i}\right|>0.01$ mdyn for $i=1, \cdots, v$, then every electron $i$ is displaced by $\bar{r}_{i} \leftarrow \bar{r}_{i}+\Delta u \bar{F}_{i} / \max |F|$, as in the program of [14], and we go to the next step of the algorithm.

Whereas, if $\max \left|F_{i}\right|<0.01$ mdyn for $i=1, \cdots, v$, the equilibrium positions of 


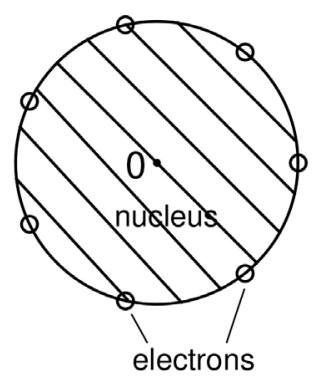

(a)

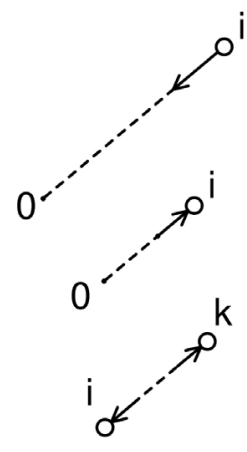

(b)

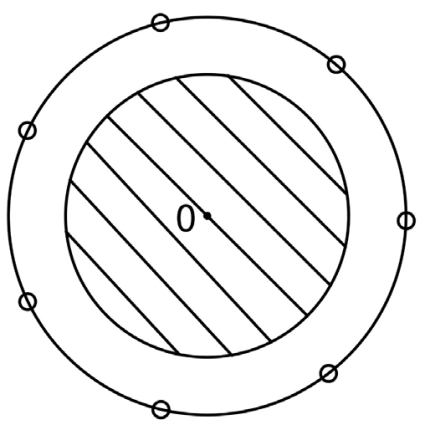

(c)

Figure 1. (a) Electrons initially located on the assumed spherical outer surface of the nucleus; (b) Attraction and repulsion from nucleus center $\mathrm{O}$ to electron $i$. Mutual repulsion between electrons $i$ and $k$, (c) All electrons equilibrate on the outer surface of the same sphere concentric and larger than nucleus.

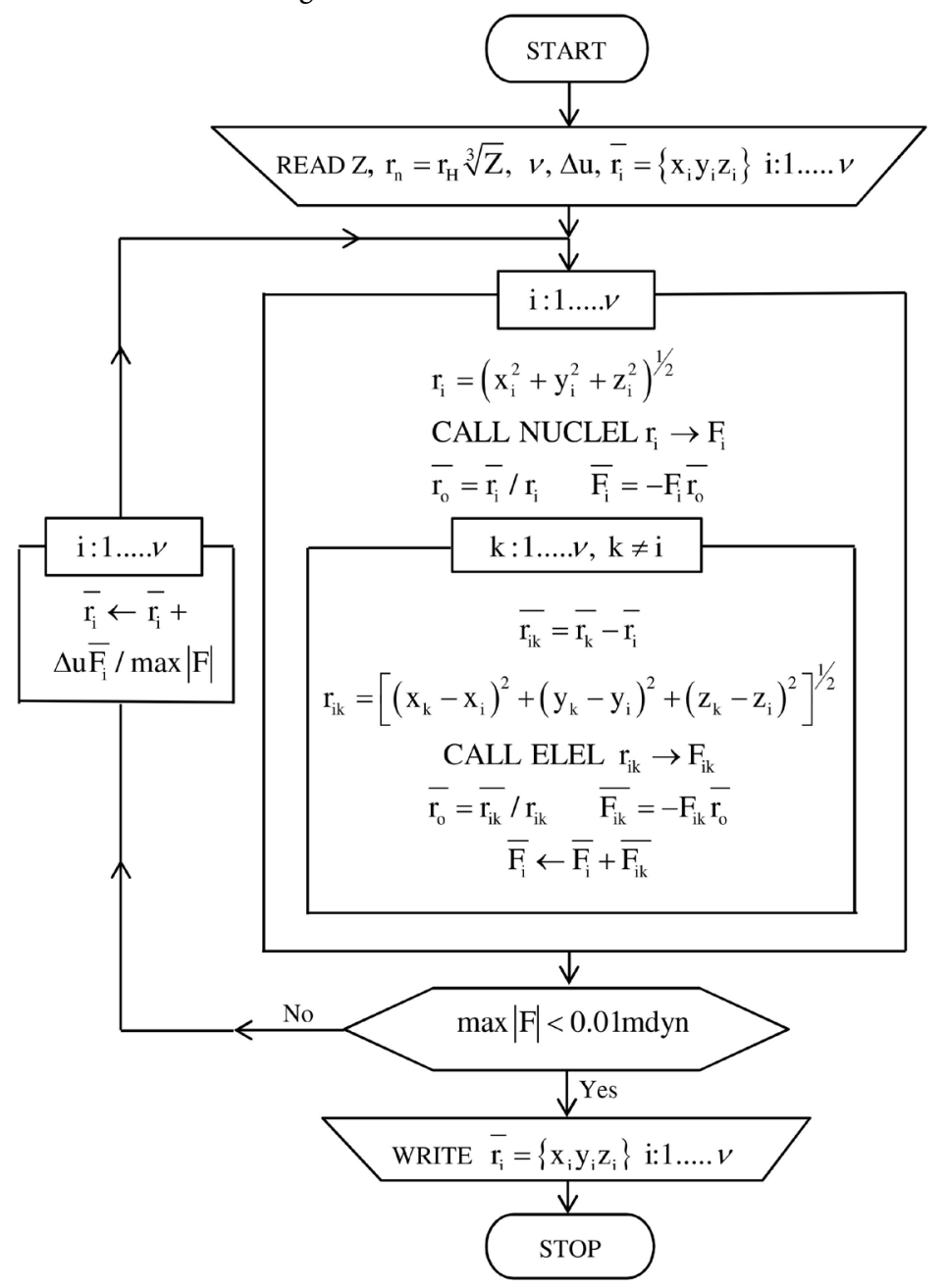

Figure 2. Flow-chart of the proposed step-by-step nonlinear static analysis algorithm for finding the equilibrium positions of electrons in an atom.

all the electrons $\overline{r_{i}}=\left\{x_{i} y_{i} z_{i}\right\}, i=1, \cdots, v$ are obtained, they are written as output, and the algorithm is interrupted. 
The program is simple and short. The main program has about 100 Fortran instructions and the subroutines, NUCLEL and ELEL, about 15 and 10 instructions, respectively. So, the program has totally about 125 instructions.

As the steplength $\Delta u$ of the algorithm is very small, e.g. $\Delta u=0.001 \mathrm{pm}$, millions of steps of the algorithm are performed; however, this happens in a few seconds of computing time.

\subsection{Estimation of Ionization Energy}

A second computer program of step-by-step nonlinear static analysis is proposed, for estimation of Ionization Energies, which can be connected to the first program of previous Section 2.1, as a continuation of it.

This second program starts from the electrons being located on the outer surface of an equilibrium sphere, as shown in Figure 3(a), and one of these electrons is subjected to gradual forced displacement, radially and outwards with respect to center $\mathrm{O}$ of nucleus. In reality, the displacement increment $\Delta u$, of this single electron, is very small; however, in Figure 3(a), it is presented with expanded scale, so that to become visible. After every forced displacement increment of this electron, we wait for the remaining electrons to equilibrate on the outer surface of a slightly smaller concentric sphere, by performing, again for them, the first program described in Section 2.1.

Within each forced displacement increment, the produced work increment is easily determined according to Figure 3(a), $\Delta E=F \times \Delta u$ and these increments

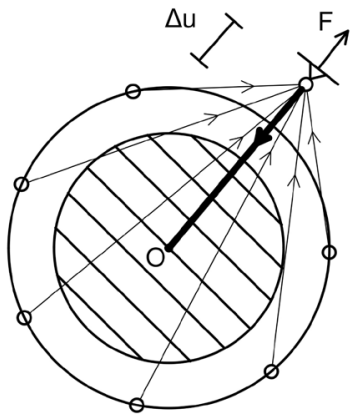

(a)

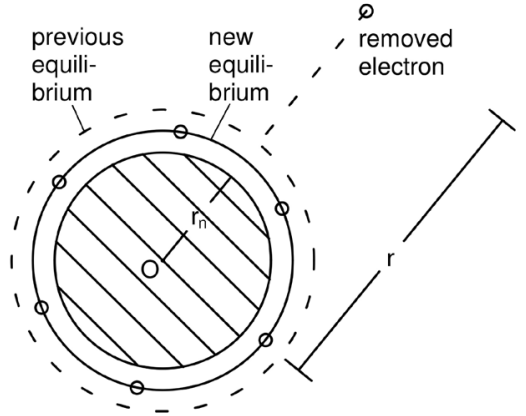

(b)

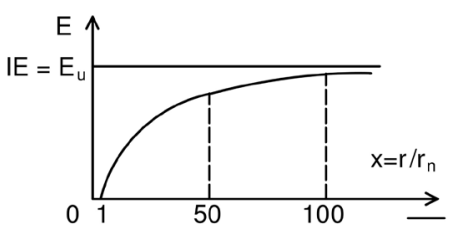

(c)

Figure 3. (a) One electron is subjected to gradual forced removal, radially and outwards, from the outer surface of an equilibrium sphere; (b) As the single electron is gradually removed, the remaining electrons equilibrate on smaller spheres; (c) The produced work of electron removal gradually increases and tends to an upper bound $E_{u}$, which is the Ionization Energy $I E=E_{u}$. 
are gradually summed $E \leftarrow E+\Delta E$, to obtain the total work $E$. As this single electron is gradually removed, the attractive force $F$ diminishes and tends to zero, $F \rightarrow 0$. So, $\Delta E \rightarrow 0$, too, thus the total work $E=\sum E_{i}$ no more significantly increases and tends to an upper bound $E_{u}$, which is the Ionization Energy $I E=E_{u}$ and the procedure is interrupted. After the removal of this single electron, the remaining electrons equilibrate on a lower shell, that is, on the outer surface of a sphere smaller than the previous equilibrium sphere, but larger than nucleus, as shown in Figure 3(b).

In Figure 3(c), is shown how the total work $E$ gradually increases and tends asymptotically to an upper bound $E_{u}$, which is the Ionization Energy, $I E=E_{u}$. This is usually achieved for $x=\frac{r}{r} \approx 100$, where $r$ is the distance of removed electron from the center $\mathrm{O}$ of nucleus and $r_{n}$ is the radius of nucleus sphere.

\subsection{Electrostatic Forces in the Atom. Mutual Repulsive Forces in a Couple of Electrons}

The mutual repulsive force $F$, in a couple of electrons, is given by the following formula, with respect to the distance $r$ between the two electrons,

$$
F=C_{p} \frac{1}{r^{2}},
$$

where the permittivity constant is $C_{p}=2.3072 \times 10^{-28} \mathrm{~J} \cdot \mathrm{m}$.

As example, let us consider the Element Argon with atomic number $Z=18$ and nuclear radius $r_{n}=97.0 \mathrm{pm}$. According to following Section 2.5, can be proved that the minimum distance between two electrons, in the proposed model, for $14<Z \leq 26$, is $\min r=97.0 \mathrm{pm}\left[0.577^{2}+2(0.707-0.577)^{2}\right]^{1 / 2}=58.77 \mathrm{pm}$

By substituting this value in Equation (1), is obtained

$$
\max F=2.3072 \times 10^{-28} \mathrm{~N} \cdot \mathrm{m}^{2} \frac{10^{24}}{58.77^{2} \mathrm{~m}^{2}}=6.680 \mathrm{mdyn},
$$

represented in Figure 4.

The derivative of function $F(r)$, with respect to $r$, according to Equation (1), which can be called stiffness $K$, is

$$
K=\mathrm{d} F / \mathrm{d} r=-2 C_{p} \frac{1}{r^{3}},
$$

and for $r=\min r=58.77 \mathrm{pm}$, gives

$$
\max K=-2 \times 2.3072 \times 10^{-28} \mathrm{~N} \cdot \mathrm{m}^{2} \frac{10^{36}}{58.77^{3} \mathrm{~m}^{3}}=-0.2273 \mathrm{mdyn} / \mathrm{pm},
$$

also shown in Figure 4.

The definite integral of function $F(r)$, from $r_{1}$ up to $\infty$, is

$$
\int_{r_{1}}^{\infty} F \mathrm{~d} r=-C_{p}\left[\frac{1}{r}\right]_{r_{1}}^{\infty}=-C_{p} \frac{1}{r_{1}}
$$

and, by referring to 1.0 mole of the Element, for Avogadro constant $C_{A}=6.022 \times 10^{23} / \mathrm{mole}$, the work produced for separation of couples of atoms 


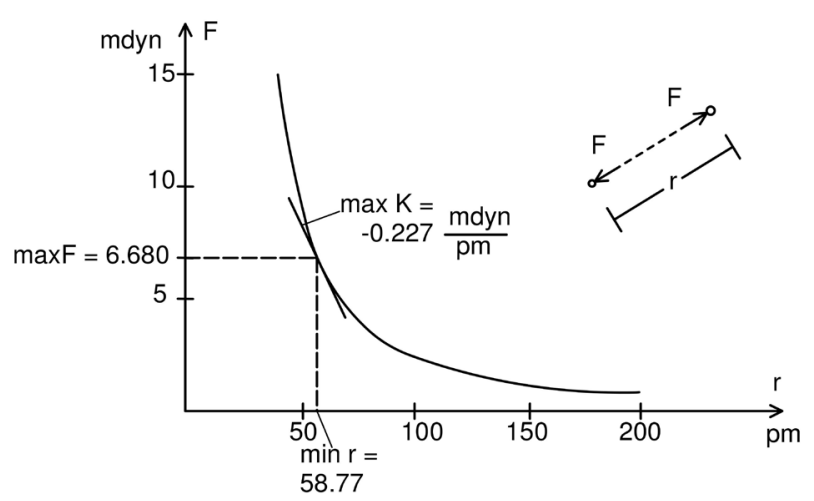

Figure 4. Mutual repulsive force $F$, in a couple of electrons, versus their distance $r$. Example: For

$\min r=58.77 \mathrm{pm}, \max F=6.680 \mathrm{mdyn}, \max K=\mathrm{d} f / \mathrm{d} r=-0.2273 \mathrm{mdyn} / \mathrm{pm}$.

from an initial mutual distance $r_{1}=200 \mathrm{pm}$ up to infinity, is:

$$
\begin{aligned}
E & =-C_{p} \frac{1}{r_{1}} C_{A}=-2.3072 \times 10^{-28} \mathrm{~J} \cdot \mathrm{m} \frac{10^{12}}{200 \mathrm{~m}} 6.022 \times 10^{23} / \mathrm{mole} \\
& =-694.7 \mathrm{~kJ} \text { oules } / \text { mole }
\end{aligned}
$$

\subsection{Lennard-Jones Curve for Nucleus-Electron Interaction (Attractive and Repulsive)}

For the nucleus-electron interaction (attractive and repulsive), a Lennard-Jones function [13] is adopted,

$$
\varphi(x)=-\frac{1}{x^{2 n}}+\frac{1}{x^{n}},
$$

where $x=r / r_{n}, r$ distance of electron from nucleus center and $r_{n}$ nuclear radius. The first above term is repulsive and the second one attractive.

In Figure 5, curves $\varphi(x)$ are shown, corresponding to the above function of Equation (3), for three different values of the parameter $n: 1.05,1.5,2.0$. It is observed, in this Figure 5, that, as the value of parameter $\mathrm{n}$ increases from 1.05 up to 2.0 , the attractive potential of the L-J function $\varphi(x)$ is gradually weakened.

The complete equation, giving the force $F$ with respect to the distance $r$, is

$$
F=Z C_{p} \frac{1}{r_{n}^{2}}\left(-\frac{1}{x^{2 n}}+\frac{1}{x^{n}}\right)
$$

where $Z$ atomic number of the Element, $C_{p}$ permittivity constant, $r_{n}$ nuclear radius and $x=r / r_{n}$. Let us try and example.

For Argon, $Z=18, r_{n}=97.0 \mathrm{pm}$, and the parameter of L-J curve, for the last four Ionization Energies ( $15^{\text {th }}$ up to $18^{\text {th }} \mathrm{IE}$ ), is $n=1.057$, as we will see in Section 3 of applications. So,

$$
F=18 \times 2.3072 \times 10^{-28} \mathrm{~N} \cdot \mathrm{m}^{2} \frac{10^{24}}{97.0^{2} \mathrm{~m}^{2}} \varphi(x)=44.138 \mathrm{mdyn}\left(-\frac{1}{x^{2.114}}+\frac{1}{x^{1.057}}\right),
$$

represented by the curve of Figure 6, where the maximum value of $\varphi(x)$ is 0.25 , so $\max F=11.035$ mdyn. 


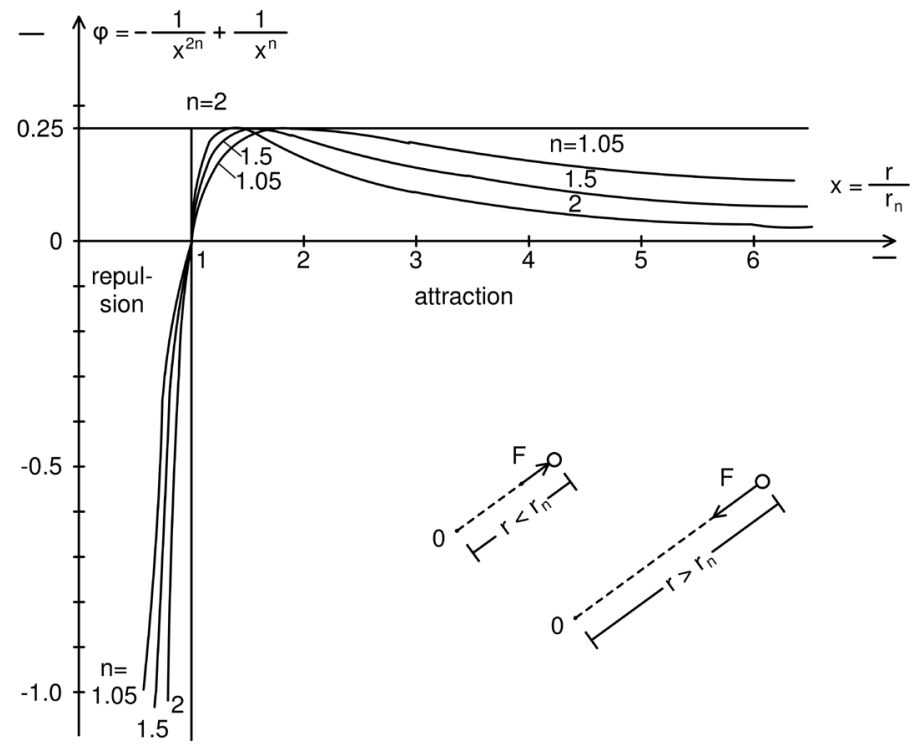

Figure 5. Lennard-Jones curves $\varphi$-X, for three values of parameter $n: 1.05,1.5,2.0$.

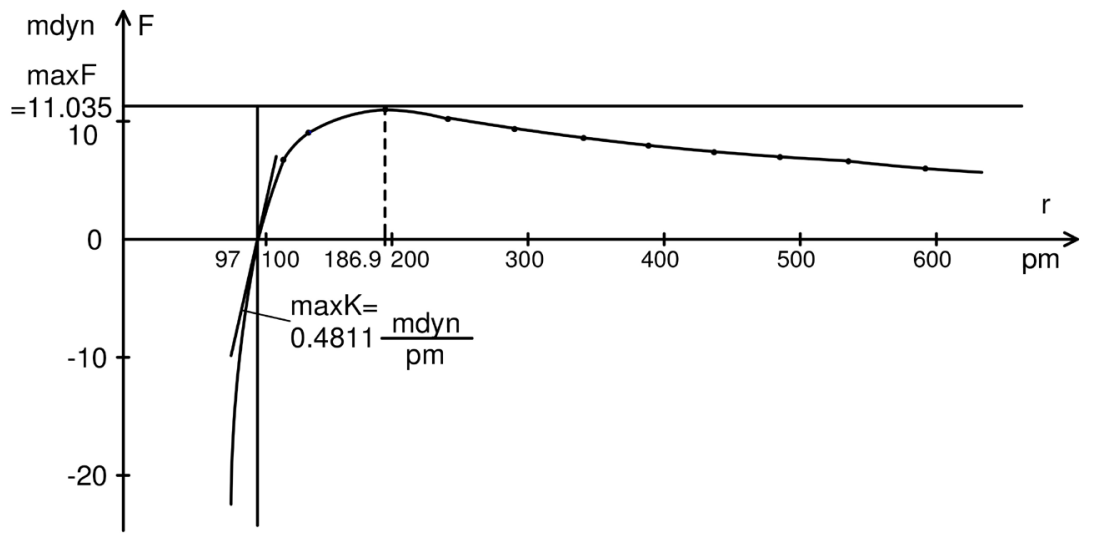

Figure 6. Nucleus-Electron curve $F-r$, for last 18th IE of Argon, with 1 electron. $Z=18$, $r_{n}=97.0 \mathrm{pm}, n=1.057$.

The derivative $\mathrm{d} F / \mathrm{d} r$ of the above function $F(r)$, which can be called stiffness $K$, is

$$
K=\frac{\mathrm{d} F}{\mathrm{~d} r}=Z C_{p} \frac{1}{r_{n}^{3}}\left(+\frac{2 n}{x^{2 n+1}}-\frac{n}{x^{n+1}}\right)
$$

and, by substituting the values of variables, for $x=r / r_{n}=1.0$, is obtained

$$
\max K=18 \times 2.3072 \times 10^{-28} \mathrm{~N} \cdot \mathrm{m}^{2} \frac{10^{36}}{97.0^{3} \mathrm{~m}^{3}} \times 1.057=0.4811 \mathrm{mdyn} / \mathrm{pm},
$$

also represented in Figure 6.

By observing the maximum values of $F$, in Figure 4 and Figure $6, \max F=$ 6.680 and 11.035 mdyn, respectively, it is obtained that an accuracy of $0.01 \mathrm{mdyn}$ is sufficient in calculations. Also, by observing the maximum stiffnesses $\max K=$ 0.2273 and $0.4811 \mathrm{mdyn} / \mathrm{pm}$, in the same Figure 4 \& Figure 6, we can choose a steplength $\Delta u$, for the step-by-step nonlinear static analysis algorithm of Section 
2.1 , according to the inequality

$$
0.4811 \mathrm{mdyn} / \mathrm{pm} \times \Delta u<0.01 \mathrm{mdyn} \Rightarrow \Delta u<0.02078 \mathrm{pm} \text {. }
$$

So, a steplength $\Delta u=0.02 \mathrm{pm}$ is sufficiently small. However, an even smaller steplength $\Delta \mathrm{u}$ can be used, up to $\Delta u=0.001 \mathrm{pm}$, for more accuracy, because millions of steps of the proposed algorithm are performed in a few seconds of computing time.

The definite integral of the L-J function $\varphi(x)$ of Equation (3) is

$$
\int_{1}^{x} \varphi \mathrm{d} x=\left(\frac{1}{n-1}-\frac{1}{2 n-1}\right)-\left[\frac{1}{(n-1) x^{n-1}}-\frac{1}{(2 n-1) x^{2 n-1}}\right]
$$

which, for $n>1.0$, exhibits an upper bound

$$
\varphi_{u}=\int_{1}^{\infty} \varphi \mathrm{d} x=\frac{1}{n-1}-\frac{1}{2 n-1} .
$$

So, in the specific example under consideration, in the last $18^{\text {th }}$ IE (Ionization Energy) of Argon, with only one electron, for the removal of this one electron in every atom of Argon, from $r_{n}$ up to infinity, for1.0 mole of Element Argon, with the accurate value of parameter $n=1.05727$, we have

$$
\varphi_{u}=\frac{1}{1.05727-1}-\frac{1}{2 \times 1.05727-1}=17.461-0.897=16.564 .
$$

And the upper bound of produced work $E_{u}$, that is the Ionization Energy, is

$$
\begin{aligned}
18 \text { th IE } & =E_{u}=Z \times C_{p} \times \frac{1}{r_{n}} \times \varphi_{u} \times C_{A} \\
& =18 \times 2.3072 \times 10^{-28} \mathrm{~J} \cdot \mathrm{m} \frac{10^{12}}{97.0 \mathrm{~m}} \times 16.564 \times 6.022 \times 10^{23} / \mathrm{mole} \\
& =427063 \approx 427066 \mathrm{kJoules} / \mathrm{mole}
\end{aligned}
$$

that is, it agrees accurately with the value of database [19].

\subsection{Rhombic Dodecahedron as Guide for Initial Locations of Electrons on Outer Spherical Surface of Nucleus}

As mentioned in Section 2.1, in the proposed step-by-step program of nonlinear static analysis, a rhombic dodecahedron [20] is used as a guide, in order to determine the initial locations of the electrons on the outer spherical surface of nucleus, so that the electrons be uniformly and sparsely arranged and avoid very small distances between neighboring electrons, which create numerical problems.

In Figure 7, is shown how a rhombic dodecahedron is formed from a regular octahedron and a cube. In Figure 8, the plan and elevation of rhombic dodecahedron are shown and the order by which, one-by-one, the electrons are added, first to the 6 vertices of regular octahedron, then to the 8 vertices of cube, projected on the circumscribed sphere and the centers of 12 faces of rhombic dodecahedron, coinciding with the middles of edges of regular octahedron, also projected on the outer surface of circumscribed sphere. 


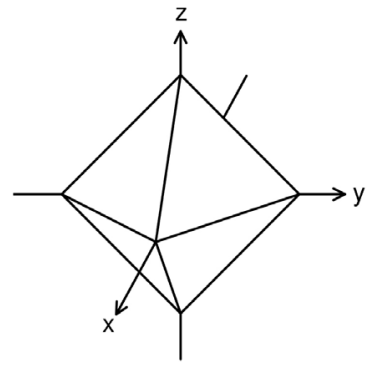

(a)

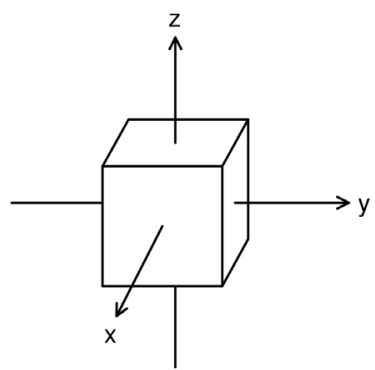

(b)

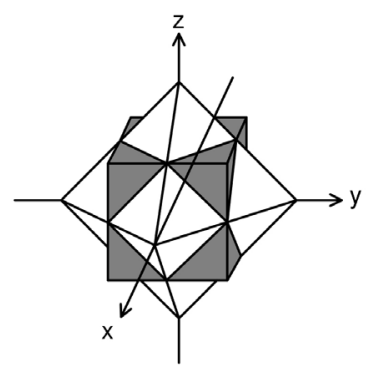

(c)

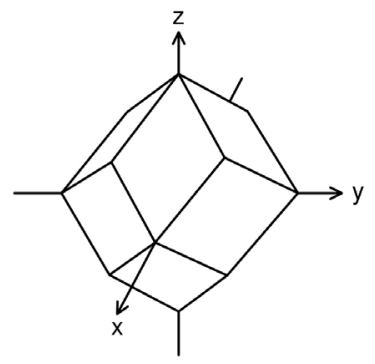

(d)

Figure 7. (a) Regular octahedron; (b) Cube; (c) Combination of regular octahedron and cube; (d) Visible faces of rhombic dodecahedron.

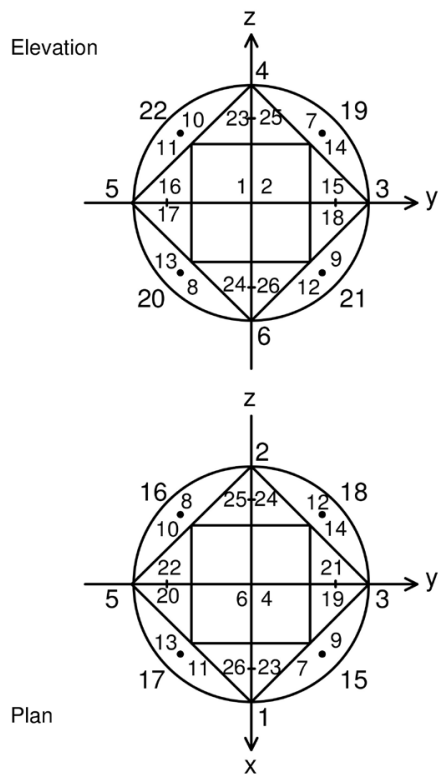

Figure 8. Plan and elevation of rhombic dodecahedron. Order by which the electrons are initially located on outer spherical surface of nucleus. Up to 26 electrons are considered here.

Attention is paid to the location of the fourth electron, so that not to lie on the same plane with the first three electrons.

In order to extend the use of rhombic dodecahedron to Elements with higher atomic numbers, $Z>26$, we can also use the $12 \times 4 / 2=24$ middles of edges, and the $12 \times 4=48$ middles of distances of every face center from the four vertices of 
corresponding rhomb. All, projected on the outer surface of circumscribed sphere, can be used as guide for initial electrons locations.

So, according to the above, the total number of electrons, which can be accommodated by the rhombic dodecahedron, is

$$
v=6+8+12+24+48=98,
$$

20 less than the 118 Electrons of the last existing Element.

In the present work, the applications will not exceed the 18 electrons. So, the rhombic dodecahedron, which is very simple, is enough here and will be used as guide for the initial locations of electrons.

\subsection{New Regular Polyhedron Accommodating Elements with High Atomic Number up to $Z=146$}

In order to accommodate Elements with very high atomic number $Z>98$, another regular polyhedron [21] is revealed, which is obtained by the projections of the 8 cube vertices on the circumscribed sphere of rhombic dodecahedron. The plan view of this regular polyhedron is shown in Figure 9(b), in comparison with the very simple plan view of corresponding rhombic dodecahedron, shown in Figure 9(a). In both Figure 9(a) \& Figure 9(b), only the visible faces are shown.

The above revealed new regular polyhedron has the 6 vertices of regular octahedron and the 8 vertices of cube, totally $6+8=14$ vertices, $6 \times 4=24$ triangular faces and $24 \times 3 / 2=36$ edges. Vertices, gravity centers of triangular faces and middles of edges, all projected on the circumscribed sphere, can be used as guide for initial electrons locations.

Also, the $24 \times 3=72$ middles of distances of gravity centers of triangular faces from corresponding triangle vertices, projected on the circumscribed sphere, can be used.

According to the above, the maximum number of electrons, which can be accommodated by the new regular polyhedron, is

$$
v=6+8+24+36+72=146,
$$

that is 28 more than the 118 electrons of last existing Element. These 146 electrons are demonstrated in Figure 10(b), in comparison with the 98 electrons that can be accommodated by the rhombic dodecahedron, shown in Figure 10(a).

\section{Case Study on Argon}

The proposed model, for finding equilibrium spheres of electrons, in an atom, and estimating Ionization Energies, will be applied on the Element Ar (Argon) [15], the noble gas of the third Period of Elements [16]. The Argon has been chosen, because complete data for Atomic Radii and Ionization Energies, for calibration and comparison, exist only for the three first Periods of the Periodic Table [17] [18] [19].

The Element Argon has atomic number $Z=18$, that is, its nucleus has 18 


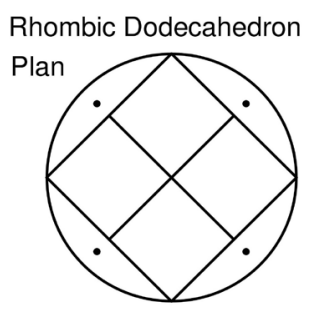

(a)

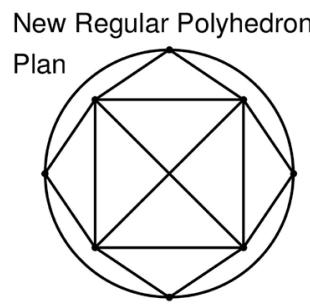

(b)

Figure 9. (a) Plan view of rhombic dodecahedron. Only visible faces. The projections of cube vertices on circum-scribed sphere are noted; (b) Plan view of the new revealed regular polyhedron. Only visible faces.

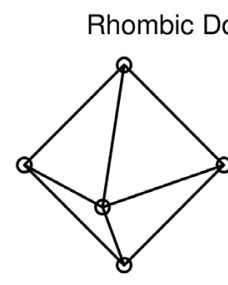

6

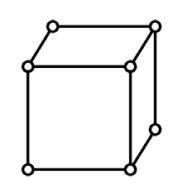

8

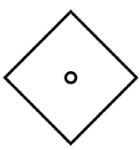

12

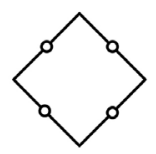

24

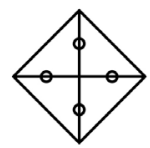

48

New Regular Polyhedron

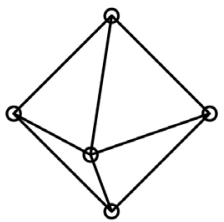

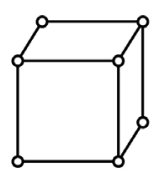

8

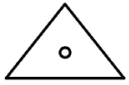

24

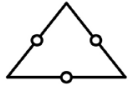

36

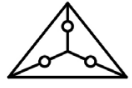

72

(b)

Figure 10. Maximum number of accommodated initial locations of electrons. (a) Rhombic dodecahedron $6+8+12+24+48=98$; (b) New regular polyhedron $6+8+24+36$ $+72=146$.

protons [1] [15]. For the radius of its nucleus, the value $r_{n}=r_{H} \sqrt[3]{Z}=37 \mathrm{pm} \times \sqrt[3]{18} \simeq 97.0 \mathrm{pm}$ is adopted, where $r_{H}=37 \mathrm{pm}$ the nuclear radius of hydrogen [1]. The above formula is reasonable, if homogeneous spherical nuclei are assumed.

In studying the IEs (Ionization Energies) of the Argon, the inverse order will be followed, that is from the last $18^{\text {th }} \mathrm{IE}$, with only one electron, by gradually adding one-by-one the electrons, up to the 1 st IE, with 18 electrons.

\subsection{Investigation of the Two Last Ionization Energies by Hand Calculator}

The two last Ionization Energies of Argon, the 18th and 17th IE, can be investigated by a hand calculator having the option of fractional exponent, on the basis of Figure 11(a) \& Figure 11(b).

For the last 18th IE (Figure 11(a)), the following formula is used, according to 


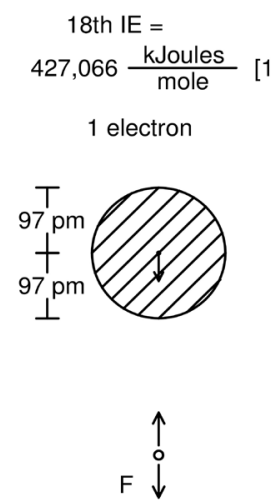

(a) 17th IE =

$397,605 \frac{\text { kJoules }}{\text { mole }}[19]$

2 electrons

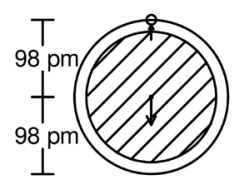

$\downarrow \uparrow$
$F \downarrow$

(b)

Figure 11. Data for investigating the two last Ionization Energies, 18th and 17th IE, of Argon.

previous Equation (6)

$$
\text { 18th IE }=E_{u}=\int_{r_{n}}^{\infty} F \mathrm{~d} r=Z C_{p} \frac{1}{r_{n}} \varphi_{u} C_{A},
$$

where $Z=18$ atomic number of Argon, $C_{p}=2.3072 \times 10^{-28} \mathrm{~J} \cdot \mathrm{m}$ permittivity constant, $r_{n}=97.0 \mathrm{pm}$ nuclear radius, $C_{A}=6.022 \times 10^{23} / \mathrm{mole}$ Avogadro constant and the upper bound $\varphi_{u}$ of L-J function integral, for parameter $n>1.0$, is,

$$
\varphi_{u}=\int_{1}^{\infty} \varphi(x) \mathrm{d} x=\frac{1}{n-1}-\frac{1}{2 n-1},
$$

according to previous Equation (5).

The database [19] gives, for the last 18th IE of Argon, the value 427,066 kJoules/mole.

By substituting the above values in Equation (7), is obtained

$$
\begin{aligned}
& 427066 \mathrm{kJoules} / \mathrm{mole}=18 \times 2.3072 \times 10^{-28} \mathrm{~J} \cdot \mathrm{m} \frac{10^{12}}{97.0 \mathrm{~m}} \varphi_{u} \times 6.022 \times 10^{23} / \mathrm{mole} \\
& \Rightarrow \varphi_{u}=\frac{1}{n-1}-\frac{1}{2 n-1}=16.654 \text { (dimensionless) }
\end{aligned}
$$

By using the diagram $\varphi_{n}-n$ of Figure 12, the value of parameter $n$, of L-J curve, is obtained graphically, for the last 18th IE of Argon, which is $n=1.05727$. In the diagram $\varphi_{n}-n$ of Figure 12(a), are found the values of parameter $\mathrm{n}$ of L-J curve, for the last IE of 12 Elements of 3 first Periods, from Argon $(Z=18)$ up to Nitrogen $(Z=7)$, all in the same way as for Argon, with help of database [19]. The same diagram $\varphi_{n}-n$ is continued to Figure 12(b), with contracted $n$ scale, where the values of parameter $n$ of L-J curve, for the last IEs of the remaining 6 Elements of 3 first Periods, from Carbon $(Z=6)$ up to Hydrogen $(Z=$ 1 ), are found in the same way.

Based on Figure 11(b), we can estimate the 17th IE of Argon, too, by subtracting, from 18th IE, the negative work produced by the separation of the two electrons, which is very small, as follows, according to Equation (2) 


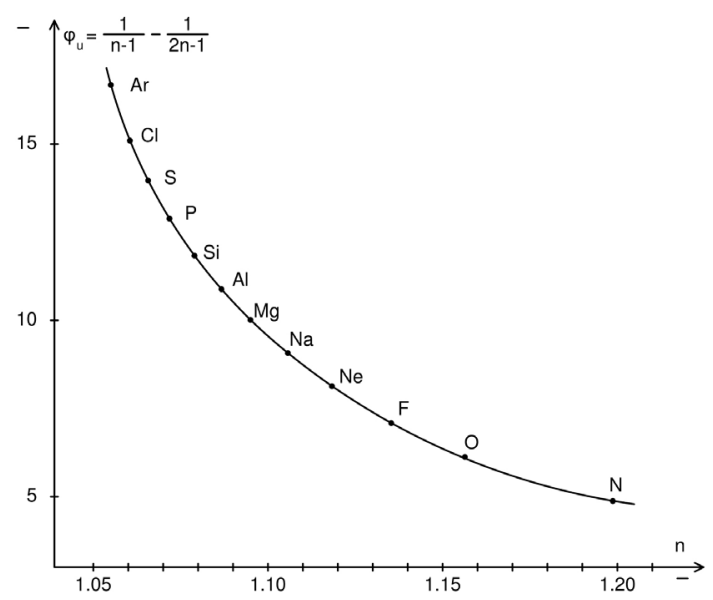

(a)

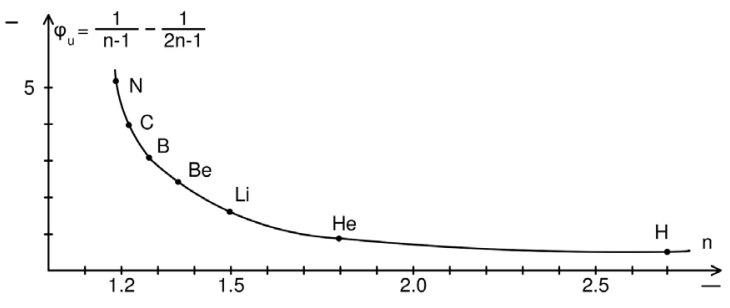

(b)

Figure 12. (a) Diagram for finding the value of parameter $n$ of L-J function from given value of its upper bound $\varphi_{u}=\frac{1}{n-1}-\frac{1}{2 n-1}$, for the last IEs of 12 Elements of 3 first Periods, from Argon $(Z=18)$ up to Nitrogen $(Z=7)$; (b) Continuation of diagram of figure (a), with contracted $n$ scale, for the remaining 6 Elements of 3 first Periods, from Carbon $(Z=6)$ up to Hydrogen $(Z=1)$.

$$
E=\int_{r_{0}}^{\infty} F \mathrm{~d} r=-C_{p} \frac{1}{r_{0}} C_{A}
$$

where $r_{0}=2 \times 98 \mathrm{pm}=196 \mathrm{pm}$, according to Figure $11(\mathrm{~b})$; permittivity $C_{p}=2.3072 \times 10^{-28} \mathrm{~J} \cdot \mathrm{m}$ and Avogadro constant $C_{A}=6.022 \times 10^{23} / \mathrm{mole}$.

By substituting these values on the above equation, is obtained

$$
E=-2.3072 \times 10^{-28} \mathrm{~J} \cdot \mathrm{m} \frac{10^{12}}{196 \mathrm{~m}} 6.022 \times 10^{23} / \mathrm{mole}=-708.9 \mathrm{kJoules} / \mathrm{mole}
$$

Thus, for the 17th IE of Argon, the value 427,066 - 708.9 = 426,357 kJoules/mole results. The database [19] gives a somewhat smaller value, 17 th $\mathrm{IE}=397,605$ kJoules/mole for Argon, which is, however close to the last 18 th $\mathrm{IE}=427,066$ kJoules/mole, too.

By substituting the value of parameter $n=1.05727$ of L-J function, found for the last 18th IE of Argon, in the Equation (4) of definite integral of L-J function

$$
\int_{1}^{x} \varphi(x) \mathrm{d} x=\left(\frac{1}{n-1}-\frac{1}{2 n-1}\right)-\left[\frac{1}{(n-1) x^{n-1}}-\frac{1}{(2 n-1) x^{2 n-1}}\right],
$$

we find how the work produced by the removal of a single electron in every 
atom, referred to 1.0 mole of the Element, varies, as the electrons are gradually removed from the atoms.

$$
\begin{aligned}
E(x) & =18 \times 2.3072 \times 10^{-28} \mathrm{~J} \cdot \mathrm{m} \frac{10^{12}}{97.0 \mathrm{~m}} \int_{1}^{x} \varphi(x) \mathrm{d} x \\
& =25783 \mathrm{kJoules} / \mathrm{mole}\left[16.564-\frac{1}{(n-1) x^{n-1}}-\frac{1}{(2 n-1) x^{2 n-1}}\right]
\end{aligned}
$$

for $n=1.05727$.

The graphical representation of the above function $E(x)$ is shown in Figure 13, for $x=r / r_{n}$ up to $10^{8}$, that is for $\mathrm{r}$ up to about $1.0 \mathrm{~cm}$. The produced work reaches up to about $280,000 \mathrm{kJoules} / \mathrm{mole}$ and tends asymptotically to the upper bound 427,066 kJoules/mole.

The produced work $E(x)$, corresponding to the 17 th IE, is slightly lower than that of last 18th IE, according to what mentioned above, and almost coincides with it.

\subsection{Running of All the Ionizations Energies of Argon by the Proposed Step-by-Step Nonlinear Static Program}

All the Ionization Energies of Argon, from last 18th IE, with 1 electron, up to first IE, with 18 electrons, have run by the proposed two step-by-step nonlinear static analysis programs of Sections 2.1, 2.2, and the results are represented in Figure 14 up to 16 , and in Table 1.

In Figure 14, according to the results of first program, the radius of equilibrium sphere varies from $r_{1}=97.0 \mathrm{pm}$, which is the nuclear radius (18th IE with 1 electron), up to $122.8 \mathrm{pm}$, which corresponds to 1 st IE, with 18 electrons $(v=18=Z)$; so, this is the atomic radius predicted by the first program. Also, an intermediate radius is shown, with value $110.4 \mathrm{pm}$, which corresponds to 10th IE with 9 electrons. More details for radii of equilibrium spheres of electrons can be found in Table 1.

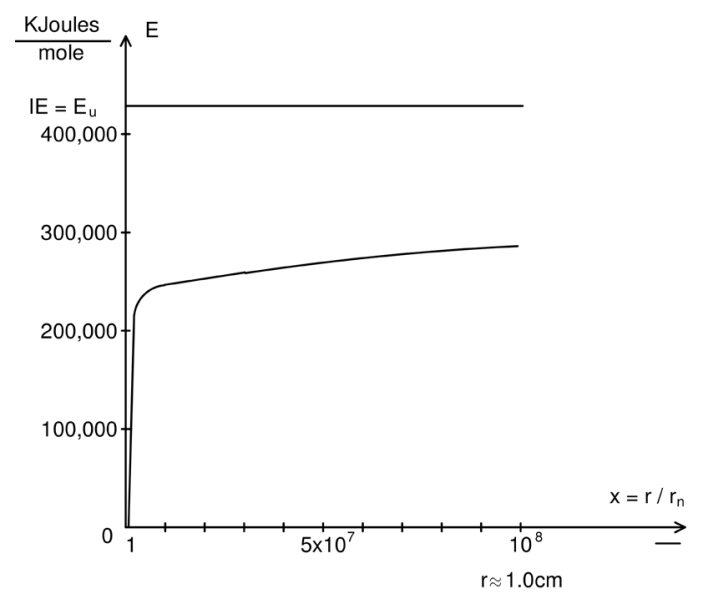

Figure 13. Variation of work produced by removal of a single electron from every atom, for one mole of the Element, corresponding to the last 18th IE, with 1 electron, of Argon, obtained by hand calculator, up to $x=r / r_{n}=10^{8}$, that is for $\mathrm{r}$ up to about $1.0 \mathrm{~cm}$. 


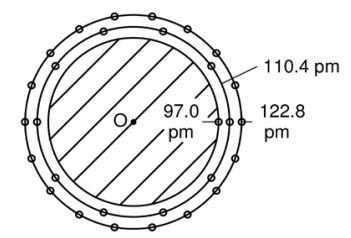

Figure 14. Gradual increase of radius $r$ of equilibrium sphere of electrons obtained by the first proposed step-by-step nonlinear static analysis program. For one electron, $v=1$, nuclear radius $r_{1}=r_{n}=97.0 \mathrm{pm}$. For $v=10$ electrons, $r_{10}=110.4 \mathrm{pm}$. For $v=18=Z$ electrons, atomic radius $r_{18}=r_{a}=122.8 \mathrm{pm}$. This value of atomic radius, found by the proposed model, is close to the average of three values of databases [15] [18], $(71+106+188) / 3=121.7 \approx 122.8 \mathrm{pm}$. As the proposed model is static, these three different values can be considered as due to dynamic oscillations around a mean static value.

Table 1. For all the IEs (Ionization Energies) of Argon, last 18th up to 1st IE, that is, for number $v$ of electrons increasing from 1 up to 18, variations of main quantities of present problem are shown: 1) Gradual increase of radius $r_{v}$ in $\mathrm{pm}$ of equilibrium sphere of electrons, from 97.0 up to $122.8 \mathrm{pm} ; 2$ ) Gradual increase of ratio of $r_{v}$ to nuclear radius $r_{n}, r_{v} / r_{n}$, from 1.0 up to $1.266 ; 3$ ) Increase of exponent $\mathrm{n}$ of L-J curve, from $n=1.057$ up to 1.93 . Between values of number of electrons $v=10$ and 11 , jump of $n$, from 1.28 to 1.68 , occurs. Increase of $\mathrm{n}$ means weakening of attractive potential of L-J curve, as mentioned in Section 2.4; 4) Decrease of IE (Ionization Energy) of database [19], from 427,066 kJoules/mole (corresponding to very large value of $x=r / r_{n}$, see Figure 13), up to 1,521 kJoules/mole. Between values of number of electrons $v=10$ and 11, abrupt fall of IE of database [19] from 40,760 to 13,842 kJoules/mole occurs; 5) Decrease of IE (Ionization Energy) of proposed model, from 81,450 up to 1542 kJoules/mole. For number of electrons between $v=10$ and 11, abrupt fall of IE of proposed model, from 39,720 to 14,020 kJoules/mole occurs.

\begin{tabular}{ccccccc}
\hline Order of IE & $\begin{array}{c}\text { Number } v \text { of } \\
\text { electrons }\end{array}$ & $\begin{array}{c}\text { Equilibrium } \\
\text { radius } r \text { in pm }\end{array}$ & $r / r_{n}$ & $\begin{array}{c}\text { L-J } \\
\text { exponent } n\end{array}$ & $\begin{array}{c}\text { Database IE } \\
\mathrm{kJ} / \mathrm{mol}\end{array}$ & $\begin{array}{c}\text { Propos. IE } \\
\mathrm{kJ} / \mathrm{mol}\end{array}$ \\
\hline 18 & 1 & 97.0 & 1.000 & 1.057 & $(427,066)$ & 81,450 \\
17 & 2 & 98.0 & 1.012 & 1.057 & $(397,605)$ & 80,750 \\
16 & 3 & 99.9 & 1.030 & 1.057 & 88,576 & 76,240 \\
15 & 4 & 101.7 & 1.048 & 1.057 & 82,473 & 76,040 \\
14 & 5 & 103.6 & 1.068 & 1.07 & 72,918 & 72,280 \\
13 & 6 & 105.1 & 1.084 & 1.10 & 66,199 & 66,210 \\
12 & 7 & 107.2 & 1.105 & 1.13 & 59,653 & 58,700 \\
11 & 8 & 108.9 & 1.123 & 1.15 & 52,002 & 50,110 \\
10 & 9 & 110.4 & 1.138 & 1.23 & 46,186 & 45,150 \\
9 & 10 & 111.9 & 1.154 & 1.28 & 40,760 & 39,720 \\
8 & 11 & 110.7 & 1.141 & 1.68 & 13,842 & 14,020 \\
7 & 12 & 111.6 & 1.151 & 1.77 & 11,995 & 10,730 \\
6 & 13 & 113.4 & 1.169 & 1.80 & 8781 & 8611 \\
5 & 14 & 114.6 & 1.181 & 1.85 & 7238 & 6473 \\
4 & 15 & 117.1 & 1.207 & 1.88 & 5771 & 4977 \\
3 & 16 & 119.6 & 1.233 & 1.90 & 3931 & 3435 \\
2 & 17 & 120.5 & 1.242 & 1.93 & 2656 & 2337 \\
1 & 18 & 122.8 & 1.266 & 1.93 & 1521 & 1542 \\
\hline & & & & & & \\
\hline
\end{tabular}


The atomic radius $122.8 \mathrm{pm}$ of Figure 14, predicted by the first program, is compared with three corresponding values of published databases [15] [18], 71, 106 and $188 \mathrm{pm}$, and found close to their average,

$(71+106+188) / 3=121.7 \approx 122.8 \mathrm{pm}$. The proposed model is static. So, the three values of databases can be considered as due to dynamic oscillations around a mean static value.

In Figure 15, obtained by the second program, is shown a group of curves representing the variation of work $E(x)$ produced by removal of one electron, referred to 1.0 mole of the Element, for $x=r / r_{n}$ ranging up to 100, that is for $\mathrm{r}$ up to about $10 \mathrm{~nm}=10^{-8} \mathrm{~m}$. Every curve corresponds to an IE, from 18th IE, with 1 electron, up to 1st IE with 18 electrons.

It is observed, in Figure 15, that, for number of electrons $10 \geq v \geq 7$, these curves $E(x)$ tend to an upper bound $E_{u}=I E$ for $x \approx 100$. Whereas, for $v \geq 11$, the curves $E(x)$ tend to an upper bound $E_{u}=I E$ earlier, for $x \approx 50$.

Particularly, it is observed, in Figure 15, that between 9th and 8th IE, that is for number of electrons $v$ between 10 and 11, an abrupt fall occurs, in these curves, with the value of $I E=E_{u}$ falling from about 40,000 to about 14,000 kJoules/mole.

In Table 1, for all the Ionization Energies, from last 18th IE with only 1 electron, up to 1st IE with 18 electrons, the corresponding values of the following quantities are given, obtained by the proposed two step-by-step programs of nonlinear static analysis and by the database [19].

1) Radius $r_{v}$, for $v=1, \cdots, 18$, of the equilibrium sphere of electrons, obtained by the first program, increasing gradually from 97.0 up to $122.8 \mathrm{pm}$.

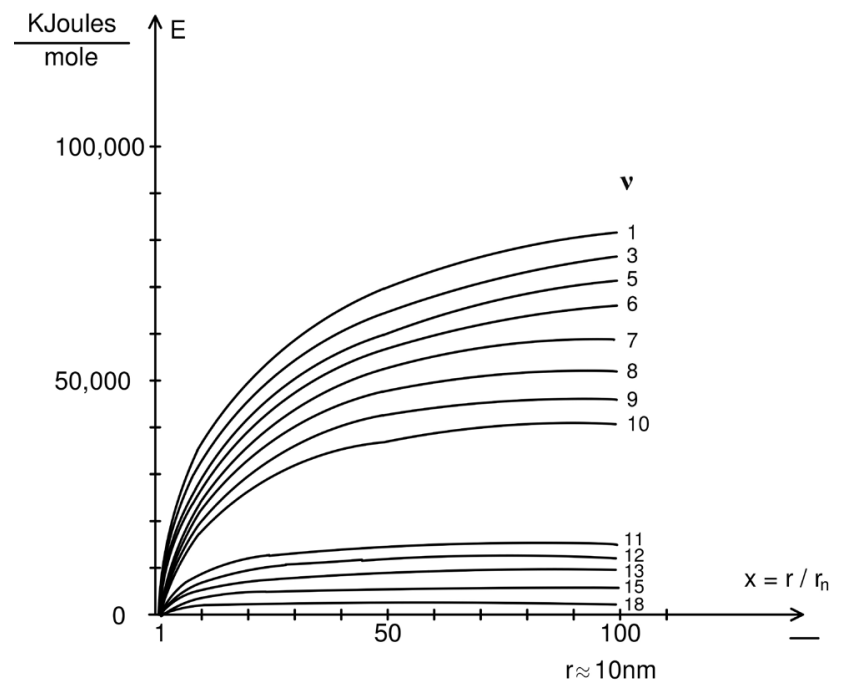

Figure 15. Variation of the work produced by removal of a single electron in every atom, for 1.0 mole of the Element Argon, from the last 18th IE with 1 electron, up to 1st IE with 18 electrons, for $x=r / r_{n}$ increasing up to 100 , that is for $\mathrm{r}$ up to about $10 \mathrm{~nm}=10^{-8} \mathrm{~m}$, where upper bound of the work $E_{u}=I E$ is reached, obtained by the second step-by-step nonlinear static analysis program. Between 9th and 8th IE, that is between $v=10$ and 11 electrons, an abrupt fall of IE occurs, from about 40,000 to about 14,000 kJoules/mole. 
2) Ratio $r_{v} / r_{n}$, for $v=1, \cdots, 18$, of radius $r_{v}$ of equilibrium sphere of electrons to nuclear radius $r_{n}$, increasing from 1.0 up to 1.266 (dimensionless).

3) Parameter $n$ of L-J (Lennard-Jones) curve (exponent of denominator of attractive term) increasing from 1.057 up to 1.93 (dimensionless). Between 9th and 8th IE, so number of electrons between 10 and 11, a jump of $n$ value, from 1.28 to 1.68 is observed. The increase, in the value of L-J parameter $n$, means weakening of the attractive potential of L-J curve, as mentioned in Section 2.4.

4) Value of IE (Ionization Energy) in kJoules/mole, obtained from database [19], varying from 427,066 up to $1521 \mathrm{kJoules} / \mathrm{mole}$. The values of two last IEs, 18th and 17th IE are very high; as mentioned previously and shown in Figure 13, they correspond to very large distances $r$ of electron from nucleus and seem not realistic. Between 9th and 8th IE, that is for number $v$ of electrons between 10 and 11, an abrupt fall of IE value of database is observed from 40,760 to $13,842 \mathrm{kJoules} / \mathrm{mole}$.

5) Value of IE (Ionization Energy) in kJoules/mole, obtained by the second proposed program, varying from 81,450 up to $1542 \mathrm{kJoules} /$ mole. In the last two IEs, 18th and 17th IE, the differences between the values of database [19] and the corresponding ones of proposed model are very large. As mentioned previously, the very large values of database, for two last IEs, correspond to very large values of removed electron distances from nucleus and seem unrealistic. For the next two IEs, 16th and 15th IE, differences, between values of database and corresponding ones of proposed model, are small. For all the other IEs, from 14th up to1st IE, that is, for number $v$ of electrons from 5 up to 18, the Ionization Energies of proposed model have been calibrated to those of database, by controlling the parameter $\mathrm{n}$ of L-J curve, so a close agreement between them is observed.

Based on the data of Table 1, the three diagrams of Figures 16(a)-(c) have been drawn:

1) Increase of radius $r$ of equilibrium sphere of electrons, from 97.0 up to $122.8 \mathrm{pm}$, for number of electrons $v=1 \ldots 18$, obtained by the first proposed step-by-step nonlinear static analysis program.

2) Increase of L-J parameter $\mathrm{n}$ from 1.057 up to 1.93 , for number of electrons $v=1, \cdots, 18$. Between $v=10$ and 11 , a jump of $\mathrm{n}$ value from 1.28 to 1.68 is observed. The increase of L-J (Lennard-Jones) parameter $\mathrm{n}$ means weakening of L-J curve attractive potential, as mentioned in Section 2.4.

3) Decrease of IE (Ionization Energy) of database [19], from 427,066 $\mathrm{kJoules} / \mathrm{mole}$ (corresponding to very large electron removal distance $\mathrm{r}$ up to 1.0 $\mathrm{cm}$, see Figure 13), up to $1521 \mathrm{kJoules} / \mathrm{mole}$, as well as decrease of IE obtained by the second proposed step-by-step static analysis program, from 81,450 up to $1542 \mathrm{kJoules} / \mathrm{mole}$ (corresponding to reasonably small electron removal distances, up to $x=r / r_{n}=100$, that is, for $\mathrm{r}$ up to about $10 \mathrm{~nm}=10^{-8} \mathrm{~m}$, as shown in Figure 15). For number $v$ of electrons between 10 and 11, an abrupt fall of IE is observed, from 40,760 to $13,842 \mathrm{kJoules} / \mathrm{mole}$ in database [19] values, and from 39,720 to $14,020 \mathrm{kJoules} / \mathrm{mole}$ in the results of second proposed step-by-step nonlinear static analysis program. 


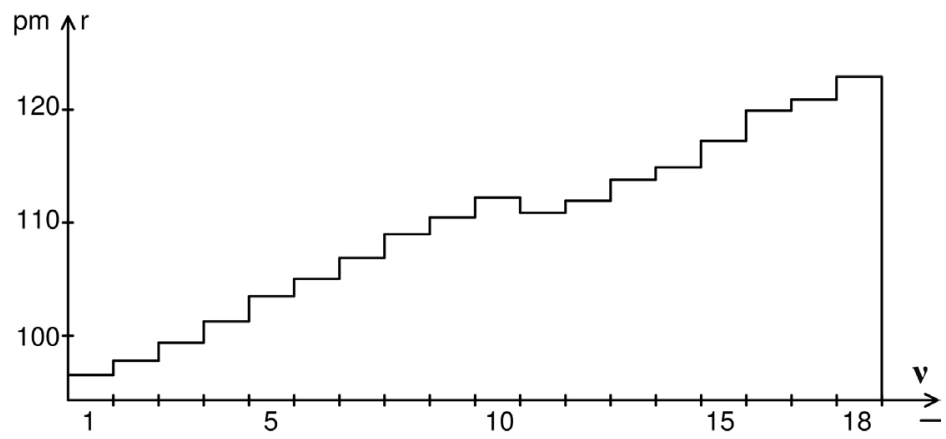

(a)

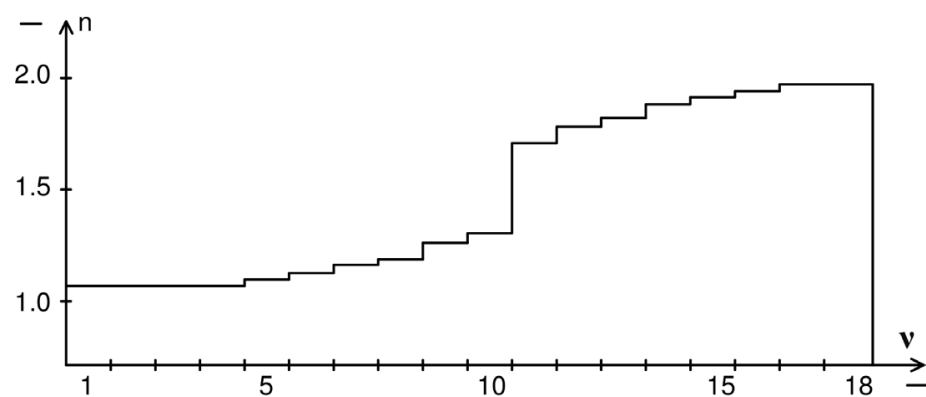

(b)

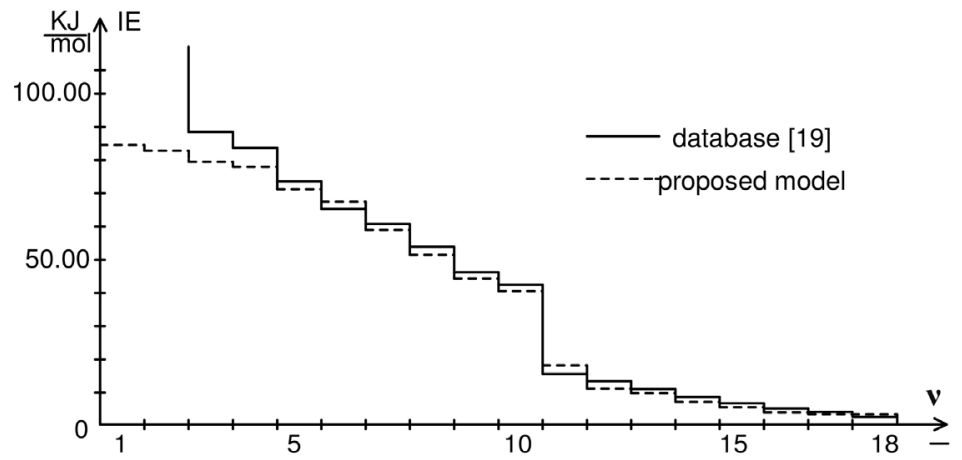

(c)

Figure 16. Based on data of Table 1, the following diagrams are obtained, for number $v$ of electrons varying from 1 up to 18, that is, for all the IEs of Argon, from last 18th up to 1st IE. (a) Gradual increase of radius $r_{v}$ of equilibrium sphere of electrons from 97.0 up to $122.8 \mathrm{pm}$; (b) Increase of parameter $n$ of L-J (Lennard-Jones) curve, from $n=1.057$ up to 1.93. Between values of number of electrons $v=10$ and 11 , jump of $\mathrm{n}$ from 1.28 to 1.68 , occurs. The increase of parameter $n$ means weakening of attractive L-J curve potential; (c) Decrease of IE (Ionization Energy) of database [19] from values about 90,000 up to about $1,500 \mathrm{kJoules} / \mathrm{mole}$. The values of last two IEs, 18th and 17th, are very high, about 400,000 kJoules, as shown in Table 1, so they are not presented in this diagram. The IEs obtained by proposed model, decreasing from about 81,000 to about $1500 \mathrm{kJoules} / \mathrm{mole}$, are also presented in this diagram c. Between values of number of electron $v=10$ and 11 , abrupt fall of IE (Ionization Energy) of database [19], as well as of proposed model, occur, from about 40,000 to about 14,000 kJoules/mole.

On the basis of three diagrams of Figure 16, the following can be observed:

As the number $v$ of electrons increases, these, by their mutual repulsive forces, are pushed to larger equilibrium spheres, which has as consequence the wea- 
kening of attractive potential of the nucleus-electron interaction, manifested by an increase of parameter $\mathrm{n}$ of L-J curve, as mentioned in Section 2.4, see Figure 5. So, the IEs (Ionization Energies) values are reduced, the electrons are more loosely connected to the nucleus and can be more easily removed.

Particularly, when the number $v$ of electrons and the radius $r$ of equilibrium sphere exceed some critical values, here $v=10$ and $r=110.0 \mathrm{pm}$ (average of two extreme values $(97.0+122.8) / 2=109.9 \mathrm{pm})$, then an abrupt fall of attractive nucleus-electron potential occurs, manifested by a jump of L-J parameter $\mathrm{n}$ from 1.28 to 1.68 , consequently an abrupt fall of IE (Ionization Energy) value, both that of database [19], as well as that of second proposed step-by-step nonlinear static analysis program, from about 40,000 to about $14,000 \mathrm{kJoules} / \mathrm{mole}$.

\section{Comparison with Published Data for First Ionization Energies and Atomic Radii of Second Period Elements}

The values of First IEs (Ionization Energies), as well as the Atomic Radii of the Elements of Second Period, Lithium $(Z=3)$ up to Neon $(Z=10)$, obtained by the proposed model, are compared with the corresponding ones from published data, based on Experiments, [1] (Chapter 7, Table 7.2, page 310, Figure 7.25, page 308). By controlling the value of L-J (Lennard-Jones) exponent $n$, from $n=$ 2.4 for Lithium, with a smooth variation, up to $n=1.915$ for Neon, a satisfactory approximation, in the values of First Ionization Energies, between published data [1] and proposed model, is achieved. Whereas, in the values of Atomic Radii, only in the first two Elements, Lithium and Beryllium, large differences are observed between published data and proposed model.

In Table 2, in the first column the symbols of Elements of Second Period are noted, from $\mathrm{Li}$ up to $\mathrm{Ne}$. In the second column, the atomic number $Z$ of the element,

Table 2. Atomic radii and first ionization energies of second period elements. 1) Element symbol. 2) Atomic number $Z$. 3) Lennard-Jones exponent $n$. 4) Nuclear radius $r_{n}$ in pm. 5) Atomic radius $r_{a}$ in pm of published data [1]. 6) Atomic radius $r_{a}$ in pm of proposed model. 7) First ionization energy in kJoules/mole of published data [1]. 8) First ionization energy in kJoules/mole of proposed model.

\begin{tabular}{|c|c|c|c|c|c|c|c|}
\hline \multirow{2}{*}{ Element } & \multirow{2}{*}{$\begin{array}{c}\text { Atomic } \\
\text { number } \\
Z\end{array}$} & \multirow{2}{*}{$\begin{array}{c}\text { L-J } \\
\text { exponent } \\
n\end{array}$} & \multirow{2}{*}{$\begin{array}{c}\text { Nuclear } \\
\text { radius } \\
r_{n} \mathrm{pm} \\
\end{array}$} & \multicolumn{2}{|c|}{ Atomic radius $r_{a}$ in pm } & \multicolumn{2}{|c|}{1 st IE in $\mathrm{kJ} / \mathrm{mol}$} \\
\hline & & & & published [1] & proposed & published [1] & proposed \\
\hline $\mathrm{Li}$ & 3 & 2.45 & 53.36 & 152 & 58.39 & 520 & 437.0 \\
\hline $\mathrm{Be}$ & 4 & 2.24 & 58.73 & 111 & 66.25 & 899 & 777.7 \\
\hline B & 5 & 2.15 & 63.27 & 88 & 72.89 & 800 & 708.4 \\
\hline $\mathrm{C}$ & 6 & 2.10 & 67.23 & 77 & 78.59 & 1086 & 989.1 \\
\hline $\mathrm{N}$ & 7 & 2.00 & 70.78 & 70 & 84.30 & 1402 & 1331.0 \\
\hline $\mathrm{O}$ & 8 & 2.00 & 74.00 & 66 & 89.12 & 1314 & 1245.0 \\
\hline $\mathrm{F}$ & 9 & 1.95 & 76.96 & 64 & 93.56 & 1680 & 1655.0 \\
\hline $\mathrm{Ne}$ & 10 & 1.915 & 79.71 & 70 & 97.80 & 2080 & 2051.0 \\
\hline
\end{tabular}


from $Z=3$ up to 10. In the third column, the L-J (Lennard-Jones) exponent $\mathrm{n}$ varying from 2.4 up to 1.915 . In the fourth column the nuclear radius obtained by the formula $r_{n}=r_{H} \sqrt[3]{Z}$, where $r_{H}=37 \mathrm{pm}$ the nuclear radius of hydrogen. In the fifth and sixth columns, the atomic radii $r_{a}$ in pm of published data [1] and proposed model, respectively. Finally, in the seventh and eighth columns, the First Ionization Energies of published data [1] and proposed model, respectively.

On the basis of Table 2, the Figure 17 has been drawn. In Figure 17(a), the variation of L-J (Lennard-Jones) exponent is shown from $n=2.4$ for Lithium up to $n=1.915$ for Neon.

In Figure 17(b), the variations of nuclear radius $r_{n}$, as well as atomic radius $r_{a}$ in pm of published data [1] and proposed model are shown, from Lithium up to Neon.

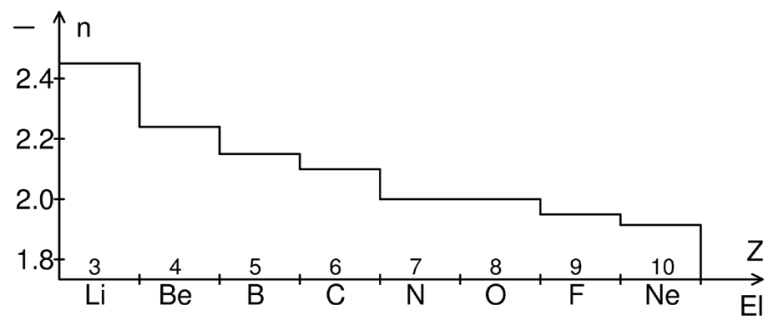

(a)

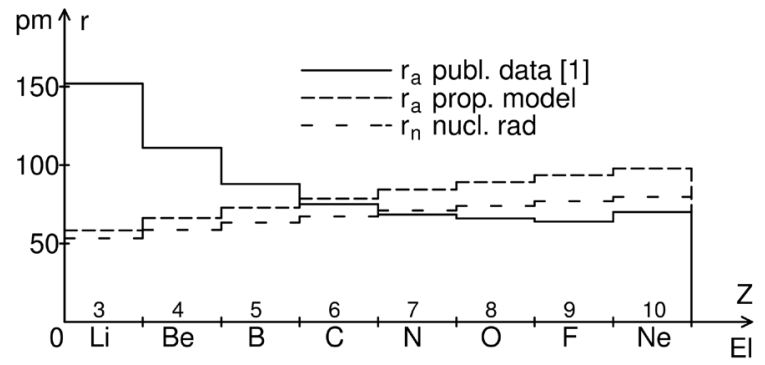

(b)

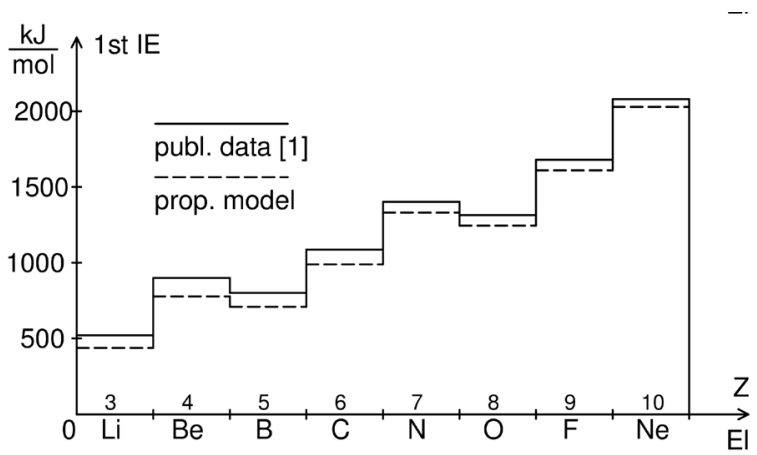

(c)

Figure 17. On the basis of Table 1, the following diagrams have been drawn, showing variations of quantities with respect to atomic number $Z$ of Second Period Elements. (a) Variation of Lennard-Jones exponent $n$; (b) Variations of nuclear radius $r_{n}$, atomic radius $r_{a}$ of published data [1], atomic radius $r_{a}$ of proposed model; (c) Variations of first ionization energies of published data [1] and proposed model. 
In Figure 17(c), the variations of First Ionization Energies in kJoules/mole of published data [1] and proposed model are shown, from Lithium up to Neon.

It is observed in Figure 17(a), that the variation of L-J exponent $n$ is smooth. The higher values of $n$ in the first Elements mean weakening of attractive potential of L-J curve, as mentioned in Section 2.4.

It is observed, in Figure 17(b), that only for the two first Elements, Lithium and Beryllium, the differences, between atomic radii $r_{a}$ of published data [1] and proposed model, are large. Before Carbon, published values of atomic radius are larger than those of proposed model. After Carbon, proposed values are larger than published ones.

In Figure 17(c), is observed, that after controlling of values of L-J exponent $n$, a satisfactory approximation, between published [1] and proposed values, has been achieved, for the first Ionization Energies of all the Elements of the Second Period. Also, in Figure 17(c), the irregularity mentioned in page 312 of [1], is observed, that is, the general trend is increase of 1st IE with increase of $Z$, with two exceptions, between $\mathrm{Be}-\mathrm{B}$ and $\mathrm{N}-\mathrm{O}$, where reduction of 1 st IE is observed.

\section{Conclusions}

1) A simplified deterministic static model is proposed investigating the equilibrium conditions of electrons in an atom and the Ionization Energies of the Elements.

2) Initially, the electrons are uniformly and sparsely arranged on the (assumed spherical) outer surface of nucleus, by using as guide a rhombic dodecahedron (in order to avoid very small distances between neighboring electrons, which create numerical problems). By taking into account nucleus-electron interactions (attractions and repulsions) and mutual electron-electron repulsions, and, by developing a simple and short step-by-step computer program of nonlinear static analysis, it is found that all the electrons equilibrate on the outer surface of the same sphere, concentric and larger than nucleus.

3) By a second step-by-step nonlinear static analysis program, connected to the first program, as a continuation of it, we start from all the electrons being in equilibrium on the outer surface of a sphere. And one of these electrons is subjected to gradual forced displacement, radially and outwards with respect to the nucleus. After each displacement increment, we wait for the remaining electrons to equilibrate on a lower shell (outer surface of a smaller concentric sphere) and the work increment, produced by the forced displacement increment, is determined. The work increments are summed. As the single electron is gradually removed from nucleus, the nucleus-electron attractive force gradually diminishes and tends to zero, so the total work, produced by removal of single electron, is no more significantly increased. The upper bound $E_{u}$, of total work of removal, is the Ionization Energy, $I E=E_{u}$.

4) For the nucleus-electron interaction (attractive and repulsive), a L-J (Lennard-Jones) type curve is adopted. When the parameter $\mathrm{n}$ of this L-J curve (ex- 
ponent of denominator of attractive term) is greater than unity, $n>1.0$, the work $E$ produced by nucleus-electron interaction, exhibits an upper bound $E_{u}$.

5) It is observed that, as the value of parameter $n$ of L-J (Lennard-Jones) curve increases from 1.0 to 2.0 (and more), the attractive potential of L-J curve is gradually weakened.

6) The proposed model is applied on Argon, the noble gas of the 3rd Period of Elements. Argon has been chosen, because, only for the 3 first Periods of Periodic Table, complete data, for Atomic Radii and Ionization Energies, exist in databases, for calibration and comparison. The atomic radius (for number of electrons $v=18=Z$ ) $r_{a}=122.8 \mathrm{pm}$, found for Argon by proposed model, is close to average of three values, given for it in databases, $(71+106+188) / 3=121.7 \approx 122.8 \mathrm{pm}$. As the proposed model is static, the three different values of databases can be considered as due to dynamic oscillations around a mean static value.

7) The IEs (Ionization Energies) of the proposed model are calibrated to the corresponding ones of databases, for Argon, by controlling the parameter $n$ of L-J (Lennard-Jones) curve, varying from value $n=1.057$ for last 18th IE with 1 electron, up to $n=1.93$ for 1 st IE with 18 electrons.

It is observed that, as the number $v$ of electrons increases from $v=1$ up to 18 , the mutual electron-electron repulsions increase, too, so the electrons are pushed to higher equilibrium shells (outer surfaces of larger concentric spheres), the radius of equilibrium sphere increases from $r_{1}=97.0 \mathrm{pm}$ (adopted for nuclear radius) up to the value $r_{18}=122.8 \mathrm{pm}$ ( $v=Z$, thus atomic radius). At same time, the parameter $n$ of L-J (Lennard-Jones) curve gradually increases from $n=$ 1.057 up to 1.93 , which means a gradual weakening of the attractive potential of L-J curve, thus the electrons are gradually more loosely connected to the nucleus and can be more easily removed; consequently, at same time, a gradual weakening of IE (Ionization Energy) occurs.

Particularly, if the number $v$ of electrons increases significantly and the radius $r$ of equilibrium sphere of electrons exceeds a critical value (in present application $110 \mathrm{pm}$, which is about average of nuclear and atomic radius $(97.0+$ 122.8)/2), then a jump (abrupt increase) of parameter $n$ of L-J curve occurs, from $n=1.28$ to 1.68 , which means an abrupt weakening of the attractive potential of L-J curve, consequently an abrupt fall of the IE (Ionization Energy), too, from about 40,000 to about 14,000 kJoules/mole, between 9th IE (10 electrons) and 8th IE (11 electrons).

8) By projecting the cube vertices of Rhombic Dodecahedron, on the outer surface of circumscribed sphere, a new Regular Polyhedron is revealed, with 14 vertices, 24 triangular faces and 36 edges, which can be used, alternatively to Rhombic Dodecahedron, as guide for initial locations of electrons, in the first proposed step-by-step nonlinear static analysis program and can accommodate Elements with high atomic number, up to $Z=146$, that is 28 more than $Z=118$ of the existing last Element. 


\section{References}

[1] Brady, J.E., Russell, J.W. and Holum, J.R. (2000) Chemistry: Matter and Its Changes. 3rd Edition, Wiley, Hoboken.

[2] Wikipedia (2018) Ionization Energy.

[3] Eloranta, Y. (2016) Undergraduate Quantum Chemistry. Department of Chemistry and Biochemistry, California State University at Northridge, Northridge.

[4] Jensen, F. (2007) Introduction to Computational Chemistry. 2nd Edition, Wiley, Hoboken.

[5] Atkins, P. and Friedman, R. (2005) Molecular Quantum Mechanics. 4th Edition, Oxford University Press, Oxford.

[6] Levine, I.N. (2009) Quantum Chemistry. 6th Edition, Prentice Hall, Upper Saddle River.

[7] Barrow, G.M. (1979) Physical Chemistry. 4th Edition, McGraw-Hill, New York.

[8] Metz, C.R. (1989) Physical Chemistry. Schaum's Outline Series, 2nd Edition, McGraw-Hill, New York.

[9] Fau, A. (2012) Finite Element Approach of Electronic Structures. Thèse, Doctorat de l' École Central (Spécialité Mécanique, Mécanique des Matériaux), Paris.

[10] Herbst, M.F. (2014) The Finite Element Method in Quantum Chemistry. Interdisziplinäres Zentrum für Wissenschaftliches Rechnen. Ruprecht Karls Universität, Heidelberg.

[11] Burt, N.J. and Dougill, J.W. (1977) Progressive Failure in a Model Heterogeneous Medium. Journal of Engineering Mechanics Division ASCE, 103, 365-376.

[12] Papadopoulos, P.G. (1984) Biaxial Network Constitutive Model. Journal of Engineering Mechanics ASCE, 110, 449-464. https://doi.org/10.1061/(ASCE)0733-9399(1984)110:3(449)

[13] Chemistry Libre Texts. Lennard-Jones Potential. https://chem.libretexts.org/Core/Physical_and_Theoretical_Chemistry/Physical_Pr operties_of_Matter/Atomic_and_Molecular_Properties/Intermolecular_Forces

[14] Papadopoulos, P.G., Koutitas, C.G. and Lazaridis, P.G. (2016) Analysis of 2D Flow by Inter-Colliding Point-Particles and Use of Incompressible Rhombic Element. Open Journal of Civil Engineering SCIRP, 6, 397-409. https://doi.org/10.4236/ojce.2016.63034

[15] Wikipedia (2018) Argon.

[16] IUPAC. International Union of Pure and Applied Chemistry. Periodic Table of Elements. https://iupac.org/what-we-do/periodic-table-of-elements/

[17] Tosto, S. (2017) Many Electrons Atoms: Ionization Energies of Transition Elements. Open Journal of Physical Chemistry SCIRP, 7, 89-121. https://doi.org/10.4236/ojpc.2017.73007

[18] Wikipedia (2018) Atomic Radii of Elements (Data Page).

[19] Wikipedia (2018) Ionization Energies of the Elements (Data Page).

[20] Wolfram Math World. Rhombic Dodecahedron. http://mathworld.wolfram.com/RhombicDodecahedron.html

[21] American Mathematical Society, Feature Column. Why Only Five? Regular Polyhedra. https://www.ams.org/publicoutreach/feature-column/fcarc-five-polyhedra 